\title{
La estética invisible del arte popular
}

\author{
ROSA FERNÁNDEZ GÓMEZ \\ Universidad de Málaga
}

\section{HACIA UN «TERCER SISTEMA DE LAS ARTES»}

EL DESCRÉDITO DEL IDEAL DE AUTONOMÍA del arte y la experiencia estética viene siendo acometido infatigablemente desde diversos frentes tanto por parte de las prácticas artísticas como de la reflexión teórica a partir de las vanguardias históricas y no debe ser grande el éxito cuando todavía surgen estudios, últimamente en clave de genealogía socio-histórica, que, al parecer, aportan algo al respecto. Uno de ellos es a mi juicio el trabajo reciente de Larry Shiner que, reconociendo su deuda con investigaciones, como las de P. O. Kristeller [1951] ${ }^{1}$ y otros, lleva el elocuente título de La invención del arte. Una historia cultural ${ }^{2}$. En este libro, de obligada lectura para todo estudiante de Bellas Artes, se sostiene que nuestra común concepción del arte es en realidad el fruto del sistema del arte del siglo XVIII en el que se establecieron una serie de divisiones, originadoras de los ámbitos opuestos del arte -las bellas artes- y la artesanía, el placer estético como goce refinado, extático e intelectualizado y el disfrute sensible común, inserto en contextos sociales y funcionales. Si bien a primera vista semejante tesis no parece contarnos nada nuevo, sin embargo, su despliegue, extensamente documentado desde la antigua Grecia hasta finales del siglo XX, nos resulta novedoso precisamente porque subraya implícitos no del todo obvios, sobre todo el hecho de que las mencionadas divisiones se establecieron en términos excluyentes con el consecuente empobrecimiento de los nuevos ámbitos, siendo este tipo de disyunción, que a menudo se nos aparece como inevitable e incluso necesaria, el producto de una suma de contingencias fácticas ${ }^{3}$.

1 Uno de los textos seminales es el artículo de Kristeller «El sistema moderno de las artes» [1951], versión castellana en: P. O. Kristeller, El pensamiento renacentista y las artes, Madrid: Taurus, 1986, pp. 179-240.

2 L. Shiner, La invención del arte. Una historia cultural, tr. E. Hyde y E. Julibert, Barcelona: Paidós, 2004. La versión original inglesa es de 2001.

3 Como bien nos muestran las tablas de verdad de la disyunción de la lógica proposicional, la oposición excluyente es sólo uno de los cuatro tipos posibles de disyunción. Más abajo me referiré al caso de la «disyunción inclusiva» propuesta por Shusterman. 
Las pérdidas se ilustran mucho mejor si atendemos a cómo se nos describe el anterior sistema del arte, -o arte / artesanía, como a menudo prefiere denominarlo el autor-, de unos dos mil años de duración, regido por una concepción del arte como construcción. Al no estar estructurado según estas divisiones excluyentes, en él, el goce podía y de hecho solía tener lugar en un contexto funcional; el nuevo, en cambio, según el modelo de la contemplación extática -que justificaría Kant con su defensa de una paradójica actitud de intenso desinterés-, exigía por parte del espectador su desligación de todo tipo de contexto. Surgía así un nuevo tipo de placer especial, que demandaba un nuevo apelativo - «estético»- y que se definiría por oposición al goce que suscita lo útil y entretenido. De entre todos los factores que desencadenaron esta dramática división, la oposición entre placer y utilidad fue, a juicio de Shiner, el más decisivo ${ }^{4}$. El siglo XIX, con el surgimiento y consolidación de instituciones como el museo, la sala de conciertos, etc., no haría más que asentar dicha oposición entre el arte y la artesanía, el placer (estético) y la utilidad, revistiéndose de rasgos espirituales y trascendentes a los primeros y denostándose aún más las labores artesanales por considerarse ahora opuestas al arte. Por su parte, los movimientos del siglo XX, a pesar de los denodados intentos de derribar fronteras, fracasaban al neutralizarse su mensaje debido a su absorción por parte del arte institucionalizado.

Formularé de modo más concreto la cuestión que aquí me propongo desarrollar citando las palabras que abren y cierran el libro de Shiner, unas reflexiones entre desengañadas y esperanzadoras pero en cualquier caso indicativas de una dirección posible para las nuevas artes que inauguran nuestro siglo XXI. En el capítulo introductorio nos confiesa la cuestión de fondo que domina todo el texto: «Mi historia de la fatal división de las artes se plantea el siguiente interrogante: ¿cómo sería el relato de las ideas e instituciones de las bellas artes si ya no lo escribiéramos como el inevitable triunfo del Arte sobre la artesanía, del Artista sobre el artesano, de lo Estético sobre la función y el placer ordinarios? ¿Por qué no escribir nuestra historia desde una perspectiva más afín a un sistema del arte que conciliase la imaginación y la destreza, el placer y el uso, la libertad y el servicio? ${ }^{5}$. Y en las líneas finales concluye apuntando en una dirección que quisiera retomar aquí: «Con este libro he intentado mostrar que las (bellas) artes, tal y como las hemos entendido generalmente,

4 En la combinación de principios que en el siglo XVIII justificaron el nuevo conjunto de artes denominadas como «bellas artes», señala Shiner: «la combinación del placer versus utilidad con genio e imaginación fue empleada para distinguir las beaux arts de las artes mecánicas o las artesanías o los oficios. La combinación de placer versus utilidad con gusto servía para distinguir las beaux arts de las ciencias y otras artes liberales, como la gramática y la lógica. Entre estos principios, el placer versus la utilidad jugaron un papel fundamental.», op. cit., p. 127.

5 L. Shiner, op.cit., p. 29. 
no son algo eterno ni antiguo, sino una construcción histórica del siglo XVIII. Además, he trazado el recorrido de una tradición paralela de resistencia que se ha prolongado hasta nuestros días. Soy consciente de que 'no hay retorno'. No podemos resucitar el viejo sistema del arte. No es posible obviar la distancia que nos separa del viejo sistema del arte atribuyendo el intelecto, la imaginación y la gracia a las bellas artes, y menospreciando la artesanía o las artes populares como dominios de la mera técnica, la utilidad, el entretenimiento y el provecho. Igual que otros dualismos que han plagado nuestra cultura, las divisiones del sistema del arte sólo pueden trascenderse mediante un esfuerzo sostenido. Creo que, desde siempre, las trascendemos efectivamente en la práctica: lo difícil es nombrar y articular nuestras prácticas» ${ }^{6}$.

¿Cuáles son las raíces de semejantes dualismos? ¿qué prácticas son esas mediante las cuales, desde siempre al parecer, nos estamos librando de la dolorosa división del arte? ¿qué tipo de experiencia nos procuran y qué proyección socio-ética y cognoscitiva podrían llegar a tener en nuestras modernas sociedades? Y más importante aún: ¿de qué manera podrían ser nombradas, articuladas, legitimadas, en definitiva? El pragmatismo americano, desde principios del siglo XX, con su énfasis en la práctica y su crítica acérrima de los dualismos procedentes de la teoría podría ayudarnos a ahondar en estas cuestiones. En efecto, fue John Dewey quien allá por 1934 publicara su célebre Art as Experience, recientemente vertido al castellano ${ }^{7}$, sosteniendo que el objeto del arte era ante todo un tipo de experiencia que tomaba como punto de partida la experiencia ordinaria de nuestra vida cotidiana -de ahí, creo, haciendo mi propia lectura de Shiner, el que siempre estemos de hecho trascendiendo las divisiones excluyentes del arte institucionalizado- . Por otra parte, entre los numerosos dualismos de nuestra cultura que denuncia a lo largo de su obra-cuerpo / mente, lo material / lo ideal, pensamiento / sentimiento, forma / sustancia, hombre / naturaleza, etc.-, habría que atribuir a la actual división entre arte refinado o elevado versus arte aplicado o popular el que en nuestras modernas sociedades no se perciba dicha continuidad entre la experiencia ordinaria y la estética, con el consecuente empobrecimiento de ambos ámbitos, el de nuestra vida cotidiana -porque satisfacemos nuestras necesidades estéticas fundamentales mediante desvíos hacia el consumo de lo vulgar- y el de nuestra experiencia del arte, porque el arte institucionalizado, deudor de las bellas artes, agoniza enclaustrado en un mundo elitista e intelectualizado sin verdadero poder comunicador ni transformador para la sociedad.

6 L. Shiner, op.cit., p. 413.

7 J. Dewey, El arte como experiencia, tr. J. Claramonte, Barcelona: Paidós, 2008. Hay una traducción anterior de S. Ramos en: México, F.C.E., 1949. 
En las líneas que siguen me propongo indagar acerca de la posibilidad de acercarnos hacia un nuevo sistema de las artes, en la expresión de Shiner, mediante la propuesta pragmatista de reconciliar lo práctico y lo estético, el arte y la vida, como modo de deshacer la radical división entre arte refinado y artesanía o trabajo productivo, placeres estéticos y placeres ordinarios, reivindicando que las labores prácticas pueden realizarse y disfrutarse estéticamente y a su vez las actividades estéticas pueden servir a fines prácticos. Para ello, en primer lugar aludiré a la crítica pragmatista del enfoque teórico al que esta corriente atribuye la visión compartimentalizada del arte y la estética; en un segundo momento, me referiré a la propuesta de funcionalidad global del arte y de la experiencia estética según Dewey, y, por último, actualizaré la cuestión planteando la posibilidad del surgimiento de nuevas prácticas artísticas a partir de la legitimación de las que de facto se dan ya en la tradición de la cultura popular, como ha sostenido un exponente destacado del pragmatismo estético en nuestros días, Richard Shusterman.

\section{De las Disecciones de las teorías-ENVOLTORIO AL PLURALISMO DISYUNTIVO-INCLUSIVO PRAGMATISTA}

Veamos un poco más en detalle el enfoque general del pragmatismo en relación con los dualismos conceptuales previamente mencionados. El punto de partida es la crítica al ideal cognoscitivo que desde Grecia ha heredado la tradición filosófica occidental de contemplación distanciada y extática, como la que tendría un espectador ante un drama, coherente con una concepción metafísica de la realidad en términos de formas estables y definidas, ordenadas racional y armónicamente. Lo sorprendente es que a pesar de que esa concepción metafísica haya quedado desfasada, sin embargo el ideal epistemológico tendente al análisis en términos excluyentes y al raciocionio compartimentalizador sigue estando vigente también en la estética analítica actual. Tanto la teoría institucional de G. Dickie como la contracrítica interpretativa de A. Danto responden al mismo modelo de «definición-envoltorio»o «teoría-envoltorio» que, basada en esquemas conceptuales clasificatorios, persigue formular una definición del arte en términos excluyentes respecto de todo lo que no constituye el arte, siendo en el caso del primero la «institución arte» y en el último la historia los contenedores para definir el arte. Este enfoque histórico es también compartido por otros teóricos recientes, como N. Carroll ${ }^{8}$, quienes al definir

$8 C f$. Una publicación reciente de Carroll en castellano que, aún defendiendo la posible dimensión moral del arte de masas, conservaría dicho enfoque compartimentalizador es: $\mathrm{N}$. Carroll, Una filosofía del arte de masas, [1998], Madrid: Antonio Machado Libros, 2002. 
el arte como práctica social o cultural logran, a juicio de Shusterman, lo mejor que cabe esperar de este modelo teórico pues, a pesar de poner el énfasis en la práctica, «al representar fielmente cómo los objetos y las actividades artísticas se identifican, se relacionan y se distinguen colectivamente, cumple del mejor modo los objetivos de definición duales de reflexión exacta y diferenciación compartimentalizada» 9 .

Frente a esta concepción de la teoría según un modelo conceptual, descriptivo y no valorativo, y frente a sus detractores por parte de posiciones pragmatistas más extremas, como las de Stanley Fish o el propio Richard Rorty que hablan de «la impotencia radical de la teoría», Shusterman defiende un pragmatismo moderado -entre posiciones de pensamiento continental europeo y corrientes analíticas anglosajonas- y sostiene una concepción de la teoría no-fundacionalista como «crítica imaginativa de la práctica» que emerge de la práctica pero a la vez tiene capacidad de intervención sobre la misma para servir a los objetivos de mejorar nuestra experiencia vital ${ }^{10}$. Este modelo de teoría pragmatista, acorde con un posicionamiento pluralista inclusivo que permite afirmar dos proposiciones alternativas, - por ejemplo la que se da entre arte refinado y popular-, mientras que no se nos convenza de que afirmar una implica negar otra, es el que propone Shusterman para acometer su proyecto de renovación del arte y la estética.

Por otra parte, y como «raíz lógica» de las mencionadas distorsiones dualistas del pensamiento occidental, que estaría en el origen último de la disociación entre estética y praxis vital, entre arte elevado y popular, me parece conveniente resaltar algo que Shusterman menciona en la segunda edición de su libro Pragmatist Aesthetics para defenderse de las críticas recibidas en la primera de 1992. Allí esgrime que todas ellas se derivan del mismo error lógico: no comprender el pluralismo de su «aserto (inclusivamente) disyuntivo»

9 R. Shusterman, Pragmatist Aesthetics. Living Beauty, Rethinking Art, Rowman \& Littlefield Publishers, Lanham, 2000, p. 43. Todas las citas de este trabajo serán una traducción propia de esta segunda edición inglesa del libro. Existe traducción al castellano: R. Shusterman, Estética pragmatista: viviendo la belleza, repensando el arte, Barcelona, Idea Books, 2002.

10 «Puesto que ninguna práctica puede definirse para toda situación posible, siempre serán necesarias proyecciones imaginativas y decisiones creativas (decisiones siempre cuestionables). Puesto que ninguna práctica existe en puro aislamiento e inafectada por otras, es necesario relacionar, coordinar o arbitrar entre distintas prácticas. En la medida en que nuestras prácticas sean así, la teoría será posible y necesaria. La teoría se revitaliza, pues, al perder su status de privilegio trascendental cognitivo. Una vez que abandonamos la visión fundacionalista de la teoría como reveladora de principios necesarios para la práctica y superamos su aspiración de justificación final y apodíptica, una vez que vemos nuestras praxis y teorías como productos contingentes cuyo encuentro con situaciones cambiantes ha necesitado continuo ajuste, clarificación, justificación y mejora, entonces, el rol de la teoría como reflexión crítica sobre la práctica está asegurado y parece ineliminable», R. Shusterman, op.cit., p. 60. 
pragmatista, según el cual, con el fin de maximizar nuestro aprovechamiento de la vida, debemos presumir que es posible afirmar conjuntamente valores alternativos hasta que no se nos convenza de que son excluyentes. Sin embargo, en el discurso filosófico occidental, ya desde Grecia, debido a que el sentido excluyente parece más preciso y dramático, esta lectura de la disyunción -presente en la mencionada distinción placer / utilidad- fue ganando una aceptación inconsciente, instaurándose el hábito de pensamiento dualista según el cual afirmar una alternativa implica negar otra ${ }^{11}$. Así, parecería que defender el arte popular, implica condenar el elevado, preocuparse por la función social del arte llevaría a rechazar los valores estéticos del mismo, o que adoptar un enfoque naturalista exigiría ignorar la constitución socio-histórica del arte, cuando en realidad, tanto él como su predecesor, Dewey, sostienen lo contrario: sólo cuando placer, conocimiento y función se dan de modo conjunto, asistimos a experiencias como la del arte.

Una vez realizadas estas aclaraciones de enfoque, habría que subrayar dos disecciones que desde el ideal gnoseológico contemplativo se han practicado ya desde la antigua Grecia, cuyas consecuencias seguimos padeciendo hoy en día y a cuya abolición se aplican los textos de estética pragmatista: la definición del arte por oposición respecto de las esferas de la realidad o la vida -heredada de la teoría mimética platónica-y su definición como práctica de hacer externo - a partir de la noción de poiesis aristotélica- que, con el tiempo, contribuiría a concebir el artefacto físico producto del arte como su objeto en vez de la propia experiencia dinámica, contribuyendo nuevamente a aislarlo de la vida y de la experiencia cotidiana, a fetichizarlo. Dewey dedicó gran parte de sus esfuerzos a combatir ambos antagonismos y para ello formuló una ambiciosa propuesta según la cual lo estético sería una cualidad aplicable a cualquier tipo de experiencia, y el arte tendría una naturaleza adjetival o modal, lo cual permitía aplicarlo de modo globalizador a cualquier dimensión, ético-práctica, socio-política o cognitiva, y a menudo a varias a la vez, de la vida humana ${ }^{12}$. A continuación ahondaré en ellas mediante el examen de cómo, según el pensamiento estético deweiano, se evidencia el falso antagonismo de las relaciones entre la experiencia estética y la ordinaria y entre las bellas artes y la artesanía o las artes utilitarias.

$11 C f$. R. Shusterman, , p. xi.

12 En El arte como experiencia leemos: «lo estético no es una intrusión ajena a la experiencia, ya sea por medio de un lujo vano o una idealidad trascendente, sino que es el desarrollo intenso y clarificado de los rasgos que pertenecen a toda experiencia completa y normal» (cit., p. 53) 


\section{LA FUNCIONALIDAD GLOBAL DEL ARTE}

El pensamiento estético de Dewey, por su enfoque holístico, histórico y socio-cultural de raíces hegelianas y en parte también deudor del idealismo de Croce, sumado a la influencia del naturalismo evolucionista, con su énfasis en la instrumentalidad práctica del conocimiento y su gnoseología falibilista, cayó en suelo estéril en el territorio intelectual norteamericano, dominado por la estética analítica y consecuentemente fue ignorado durante un largo tiempo ${ }^{13}$. Sin embargo, desde la década de los ochenta y sobre todo los noventa, con el auge de los estudios culturales y de la interdisciplinariedad en las artes, su pensamiento cobra una vigencia renovada.

$\mathrm{Al}$ margen de que comulguemos o no con el enfoque general de su pensamiento, es innegable que El arte como experiencia resulta increíblemente avanzado para su tiempo al diagnosticar ya un estado de crisis en las artes que no ha dejado de abandonar a todo el arte posterior. Tal y como él lo entendía, la cuestión más acuciante era la profunda escisión existente en su tiempo entre el arte refinado, - «la concepción museo del arte»-y la experiencia cotidiana, un abismo que le impedía a la gente sencilla concebir que aquello que les ayudaba a entretenerse, el cine comercial, el jazz, las noticias de los periódicos o los comics, escondiese en su disfrute motivaciones de índole estética. Además de las raíces filosóficas profundas previamente apuntadas, los motivos históricos concretos a los que atribuía dicha separación estaban relacionados con el advenimiento del capitalismo, los nacionalismos e imperialismos ${ }^{14}$, posicionándose, así, en un lineamiento cercano al marxismo de la escuela de Frankfurt, sobre todo a Benjamin, por su defensa de la democratización del arte, aunque el enfoque de la teoría crítica difiera en gran medida de la inspiración deweiana.

Precisamente, lo más interesante de su aportación, a mi entender, proviene de lo singular de su enfoque pragmatista como conglomerado de las más variadas influencias que culmina en su propuesta del enraizamiento de la experiencia estética y el arte en la vida cotidiana, lo que se ha dado en llamar «la tesis de la continuidad», que él define en clave de problema: «recobrar la continuidad de la experiencia estética con los procesos normales de la vida» ${ }^{15}$. Para Dewey, el arte y la belleza están en cualquier dimensión de la vida, ante todo porque responden a necesidades biológicas básicas que el «organismo humano» com-

13 La principal crítica que ha recibido su obra desde posturas analíticas e incluso desde otras más afines es la vaguedad de su noción de experiencia estética y el poco poder explicativo que se le atribuye dado que el mismo autor reconoce que se trata de una experiencia inefable. Shusterman, aunque parcialmente de acuerdo en este punto con sus críticos, esgrimirá que se le juzga desde un paradigma epistemológico equivocado.

$14 C f$. J. Dewey, op.cit., cap. 1

15 J. Dewey, cit., p. 11. 
parte con los animales; el arte es un resultado de la interacción del organismo vivo y su entorno y su función principal es la de contribuir a la integración de nuestras dimensiones corporales e intelectuales, de ahí que su enfoque haya sido denominado «naturalismo somático».

Por ello, para indagar en lo estético hemos de huir del museo y de las obras canonizadas y salir a la calle a observar los placeres comunes de nuestros congéneres mientras realizan labores cotidianas gozosamente absortos en su quehacer, desde el ama de casa que se afana en regar las plantas, el hombre que atiza el fuego, el que planta un manto de césped, hasta incluso el mecánico. Sobre este último afirma Dewey en un célebre pasaje: «el mecánico inteligente, comprometido con su trabajo, interesado en hacerlo bien y que encuentra satisfacción en su labor manual, tratando con afecto genuino sus materiales y herramientas, está comprometido artísticamente» ${ }^{16}$.

Desde este enfoque holístico o globalizador, al concebirse el arte y su experiencia como una cualidad de la experiencia ordinaria, se le otorga al arte el más alto valor funcional, siendo la tradición kantiana la concepción más antitética a la que se enfrenta Dewey en su obra. El error de ésta última provendría de asumir, a partir de premisas teóricas dualistas, que puesto que en la modernidad el arte ya no tiene ningún fin o función específica que pueda realizar mejor que ningún otro ámbito, su legitimación sólo puede venir de su emplazamiento al margen de todo uso y funcionalidad, esto es, teniendo un fin y funcionalidad intrínsecos -el placer altamente intelectualizado que se corresponde con la finalidad formal de la Crítica del Juicio-. Esta confusión, conviene subrayarlo aquí, suele basarse en un supuesto antagonismo medios / fines, en la idea de que lo instrumental se opone a lo que tiene valor intrínseco, algo que el pragmatismo estético cuestiona abiertamente. En palabras de Shusterman: «la presunta oposición práctica / estética descansa sobre la equivocada construcción de una distinción funcional entre medios y fines entendida fundamentalmente como una división en términos de oposición entre ambos. Confundir los medios con meras condiciones causales externas y coercitivas para un fin implica asumir falsamente que lo que funciona como medio no puede a la vez ser elegido libremente y disfrutado como un fin; del mismo modo, se yerra al no reconocer que los medios pueden incluso saborearse como un aspecto que contribuye al fin respecto del cual son medios» ${ }^{17}$.

Dewey, en concordancia con esta no-distinción radical entre funcionalidad instrumental y valor intrínseco, sostuvo que la funcionalidad propia del arte no radicaba en ningún fin particular y especializado sino en satisfacer a la «criatura viva» de modo global, siendo a la vez algo valioso instrumentalmente y un fin

16 J. Dewey, cit., p. 6.

17 R. Shusterman, op.cit., p. 50. 
que satisface por sí mismo; si lo que es una utilidad satisface un fin limitado, la obra de arte estética satisface muchos fines al servir a la vida en vez de prescribir un modo de vida definido y limitado. Sobre la distinción entre bellas artes y artes útiles advierte: «Es habitual y necesario desde algunos puntos de vista, hacer una distinción entre las bellas artes y las artes útiles o tecnológicas. Sin embargo, el punto de vista desde el cual es necesario hacerla es extrínseco a la obra de arte misma. La distinción más común está basada simplemente en la aceptación de ciertas condiciones sociales existentes. Supongo que los fetiches del escultor negro eran considerados de gran utilidad por el grupo tribal, más aún que las lanzas y los vestidos. Ahora son bellas artes, que sirven, en el siglo $\mathrm{XX}$, para inspirar renovaciones de las artes que se han hecho convencionales. No obstante, son bellas artes únicamente porque el artista anónimo vivió y experimentó plenamente, durante el proceso de producción. (...) Este grado de compleción al vivir la experiencia de hacer y de percibir es lo que constituye la diferencia entre lo que es bello o estético en el arte y lo que no lo es. El hecho de que lo producido se usa como tazas, capas, adornos, armas, nos resulta, hablando intrínsecamente, del todo indiferente» ${ }^{18}$.

La experiencia estética contribuye a mejorar y profundizar en nuestras demás actividades, mientras que el arte tiene el poder de hacernos experimentar el mundo común en su plenitud al conferirle una unidad dinámica a nuestra experiencia que la intensifica y realza. Ambos, como dos momentos de un mismo proceso, no se distinguen, pues, en términos clasificatorios por rasgos únicos y exclusivos, sino por su capacidad de integrar y llevar a su consumación más plena nuestras experiencias normales, -lo que Dewey denominaba «tener una experiencia»-, constituyendo una totalidad significativa vital a partir de su variedad y otorgándole a la persona que la experimenta un sentimiento de plenitud y orden en el mundo.

\section{LA LEGITIMIDAD ESTÉTICA DEL ARTE POPULAR}

El pensamiento estético de Shusterman es una continuación, pero sobre todo una aplicación práctica y concreta de los lineamientos tan vastos que marcaba Dewey. Por ello, a diferencia del primero -que, por otra parte, no se

18 J. Dewey, cit., p. 31. Su distinción entre lo extrínseco e intrínseco también lo expone al reflexionar sobre los medios en el arte: «siempre que medios y fines son externos recíprocamente, son no estéticos. Esta exterioridad puede aún ser considerada como una definición de lo no estético (...) Cuando los griegos identificaban lo bueno y lo bello en la acción, revelaban en su sentimiento de gracia y proporción en la conducta recta, una percepción de la fusión de medios y fines», op.cit., p. 223. 
caracterizaba especialmente por defender las artes populares de su tiempo-, éste sí cree útil y necesario realizar, a efectos prácticos, ciertas clasificaciones y por ello defenderá en una serie de escritos que le han hecho célebre el rap, el rock o la música country como formas artísticas igualmente legítimas estéticamente que el fine art ${ }^{19}$.

«La razón más poderosa y urgente para defender el arte popular es que nos proporciona (incluso a nosotros los intelectuales) demasiada satisfacción estética como para aceptar su total rechazo por inmoral, deshumanizador y estéticamente ilegítimo. Condenarlo como apropiado sólo para el gusto bárbaro y el embotado entendimiento de las no-iluminadas masas manipuladas es dividirnos no sólo contra el resto de nuestra comunidad sino contra nosotros mismos. Estamos hechos para desdeñar las cosas que nos dan placer y para sentirnos avergonzados del placer que nos procuran ${ }^{20}$. En estos provocativos términos formula Shusterman su apología del arte popular, apuntando sus dardos contra lo que él considera el mismo corazón dividido de la comunidad académica e intelectual a la que él pertenece, situando de paso todo el discurso estético de crítica al arte popular en la estela de renuncia ascética que se iniciara con Platón y que nos ha conducido a establecer una rígida línea divisoria entre la cultura elevada y la popular; algo que en última instancia, como ya señalara Nietzsche, refleja el miedo a la vida sobre el que se ha construido la superestructura ideológica de nuestra tradición occidental. Y no es que se trate de abolir la institución arte, muy al contrario, se trata de transformarla en el sentido de que se redefina a partir del cuestionamiento del actual antagonismo entre lo elevado y lo popular; tampoco de negar su autonomía, pero sí que esta se fundamente en términos de total diferenciación respecto de la praxis vital. Para ello, un primer paso sería justificar la legitimación estética del arte popular, lo cual afectaría a la propia concepción del arte elevado, actualmente definido por oposición a la cultura popular.

Uno de los argumentos que a primera vista nos podría convencer de la necesidad de revindicar el arte popular es el fracaso que ha supuesto los intentos de las propias vanguardias de subvertir el establishment burgués mediante el cuestionamiento de la autonomía del arte elevado y la aproximación de las prácticas artísticas a la vida cotidiana. Sobre los esfuerzos del Dadá y ciertas prácticas del performance art reciente comenta Shusterman que, al par que la institución del arte elevado los desarma «estéticamente» y se lo reapropia, chocan y confunden de modo engañoso a la población «culturalmente dominada», teniendo la incomprensión que estas obras producen, el efecto perverso de

19 R. Shusterman, op.cit., caps. 7 y 8 y también su Performing Live. Aesthetics Alternatives for the End of Art, Ithaca, New York: Cornell University Press, 2000, cap. 4.

20 R. Shusterman, op.cit., p. 170 
reforzar en ella el sentimiento de inferioridad y la creencia de que su exclusión de la cultura elevada está justificada. Por ello, cree Shusterman que la crítica de arte y la teoría estética, al haber estado durante tanto tiempo alimentados por la ideología del arte elevado, quedan neutralizados como instancias a partir de las cuales producir la reintegración del arte en la praxis de la vida ordinaria. De ahí el papel crucial del arte popular como alternativa: «el arte popular (...) podría ser una fuerza prometedora para transformar nuestro concepto e instituciones del arte hacia una mayor libertad y una integración más cercana a la praxis de la vida ${ }^{21}$.

Ahora bien, aunque podríamos analizar ejemplos concretos, y Shusterman lo hace, de obras de artes populares actuales -en su mayoría procedente de la cultura de los mass-media y las nuevas tecnologías, de los ámbitos del cine comercial, los dramas y comedias televisivos, la música rock, el rap, el country-que reúnen requisitos para ser legitimadas estéticamente, es innegable que, en su estado actual, la mayor parte de este tipo de producciones culturales sufre un grave estado de degradación debido precisamente -y en esto Shusterman se confiesa seguidor de Dewey- a su exclusión del dominio del arte autónomo, algo que en última instancia es fruto de la profunda compartimentalización de esferas de nuestras modernas sociedades ${ }^{22}$. En alusión a un célebre poema de T. S. Eliot, Portrait of a Lady, donde se relata la relación entre un joven y una vieja dama, y que Shusterman interpreta como la relación que se podría dar entre el arte popular y el elevado, comenta: «Exiliado de la respetabilidad estética y la aceptación de nuestra tradición artística, el arte popular es desprovisto del cuidado y control artístico que podría hacerlo más satisfactorio y sensible estéticamente. Abandonado a deformarse debido a las presiones de la vida, que son en gran medida las presiones deshumanizadoras del beneficio económico, crece robusto, técnicamente sofisticado, pero brutalmente crudo en sensibilidad. Por el contrario, al arte elevado, como a la dama, se le deja morir solo en su sofocante pureza y espiritualidad sin vida, divorciado de las vigorizantes energías de la vida (la schilleriana «belleza enérgica») que pertenecen a las formas expresivas populares tanto como al joven» ${ }^{23}$. El antagonismo, tiene, pues, repercusiones negativas para ambos ámbitos y nos enfrenta con

21 R. Shusterman, op.cit., p. 145.

22 El pensamiento de autores como Adorno y otros seguidores de la escuela de Frankfurt, que sostendrán el aislamiento del arte elevado como requisito para ejercer desde una supuesta e incontaminada autonomía estética su función crítica de la sociedad moderna fragmentada, aparecen a la luz del pragmatismo como últimamente vinculados con el ideal platónico ascético de renuncia y negación de la vida, deudores de una concepción del placer sumamente intelectualizado y negador de nuestro componente somático, del cual parte, en última instancia nuestras dimensiones artístico-estéticas.

23 R. Shusterman, op.cit., p. 168. 
un dilema estético que se podría solucionar cuestionando las mismas bases filosóficas de tal división.

Shusterman admite, pues, que la mayoría de los productos del arte popular actual carecen de total interés estético y tienen efectos sociales muy perniciosos cuando son consumidos de modo pasivo y sin cuestionamiento alguno. Con su planteamiento, que denomina meliorista, lo que pretende poner en entredicho son los argumentos filosóficos que, a partir de esta situación de hecho, infieren que todo arte popular deba ser siempre y necesariamente estéticamente inaceptable. El meliorismo defiende que el arte popular debería ser mejorado porque de hecho puede serlo y, de este modo se sitúa entre el pesimismo condenatorio de los elitistas de la alta cultura y del marxismo de la escuela de Frankfurt y el optimismo laudatorio procedente de instituciones conocidas en América como la Popular Culture Association y el Journal of Popular Culture ${ }^{24}$.

Para demostrar el estatuto estético del arte popular, Shusterman se vale tanto de la argumentación filosófica general como del análisis concreto de formas de arte popular, con atención específica a obras concretas, pretendiendo con ello demostrar que «el arte popular no sólo puede satisfacer los estándares estéticos más importantes de nuestra tradición estética, sino que también tiene el poder de enriquecer y reformular nuestro concepto tradicional de lo estético, de modo que se lo libere más plenamente de su asociación alienante con el privilegio de clase, la inacción socio-política y la negación ascética de la vida» ${ }^{25}$.

\section{REPARAR FISURAS}

Para finalizar, quisiera retomar la problemática arte $v s$. artesanía, placer $v s$. utilidad, desarrollada por Shiner y planteada al inicio de mi reflexión, y relacionarla con el modo en el que Shusterman trata de defender, a partir de los planteamientos globales deweianos, pero también de los suyos propios, el arte popular frente al elevado.

Si bien, como he tratado de mostrar, el antagonismo popular / elevado, estético / práctico tiene una honda raigambre filosófica griega, que aún hoy perdura en esa especie de negación ascética de la vida que subyace en la obra de pensadores de muy diverso cuño, no es menos cierto que es precisamente en los albores de la modernidad dieciochesca y no antes, cuando esos gérmenes florecen, llevándose a pleno cumplimiento sólo en los siglos XIX y XX, coincidiendo con la fundación y desarrollo de la estética como disciplina filosófica. A veces, parecería que Shusterman pasa un poco por alto este hecho

24 R. Shusterman, op.cit., p. 176.

25 R. Shusterman, op.cit., p. 173. 
cuando trata de defender la legitimidad estética del arte popular intentando mostrar que cumple con los criterios que definen lo estético tal y como ha sido planteado a partir de la constitución moderna de la disciplina; esto es, cuando a través de complejas y agudas argumentaciones trata de convencernos de que, al igual que el arte elevado, el popular también procura una prolongada satisfacción estética y permite la innovación creativa, la experimentación en la forma, la exploración de profundas cuestiones sociales, políticas o filosóficas y es susceptible de ser entendido a varios niveles ${ }^{26}$; es decir, trata de legitimarlo a partir de los criterios que, por ser el resultado de la división del arte, deberíamos evitar pues implican ya la escisión de lo elevado y lo popular.

Al defender el fine art of rap, sin embargo, lo que nos sirve para reivindicar la legitimidad estética de las obras, son rasgos postmodernos tales como la apropiación recicladora en vez de la creación de origen único, la mezcla ecléctica de estilos, abrazar las nuevas tecnologías de la cultura de masas, el cuestionamiento de la autonomía estética modernista y de la pureza artística y el énfasis en lo local y temporal en vez de lo universal y eterno ${ }^{27}$.

A mi entender estos criterios postmodernos, al poner en entredicho los ideales de la modernidad, son más acordes y coherentes con una defensa de las artes populares, pero además, si aceptamos las premisas de Shiner, como modo de enriquecer y profundizar en los criterios que podrían validar unas nuevas artes no-divididas, podríamos valernos de la inspiración procedente de toda la tradición pre-moderna. De este modo, si en este sistema del arte como construcción al artesano / artista se le exigía una combinación de genio y aplicación de reglas, inspiración y facilidad, innovación e imitación, libertad y servicio, bueno sería, para un arte más integrado en la praxis vital, que se tratase de fomentar desde las instituciones volver a integrar esas cualidades ideales que una vez estuvieron unidas o al menos no del todo desunidas.

Un primer paso en la dirección de un nuevo sistema de las artes podría ser ayudar a las generaciones venideras a comprender, como nos hace ver Shiner ${ }^{28}$, las raíces genealógicas dualistas y excluyentes de rasgos que, desde el siglo XVIII venimos atribuyendo incuestionadamente al artista, tales como: 1. la originalidad, porque se construye por oposición al principio de la mimesis, 2. la inspiración o entusiasmo y el énfasis en la expresión, porque se lo entiende como algo irracional opuesto a la aplicación de reglas, 3. la imaginación, porque al concebirse creativamente, implica la idea de creación como fin en sí mismo, 4. la creación, porque del mismo modo se la entiende como un fin en sí mismo opuesto a la invención en el sentido de descubrimiento de lo previamente

$26 C f$. R. Shusterman, cit., cap. 7.

27 R. Shusterman, p. 202.

28 L. Shiner, cit., pp. 164-69. 
dado, etc.. Sólo así podríamos empezar a vislumbrar la continuidad estética que nos permitiese ensanchar las fronteras del hecho artístico hasta abarcar los fenómenos más íntimos y cercanos de nuestra experiencia cotidiana.

Además, de modo complementario, proponer como alternativa a dicho sistema del arte dividido, una concepción del arte y la estética en términos pragmatistas, esto es, profundamente imbricados en la vida de las personas e impregnados holísticamente de las dimensiones éticas, sociales y cognoscitivas que nos conforman como seres humanos, ha sido el cometido de estas líneas. 